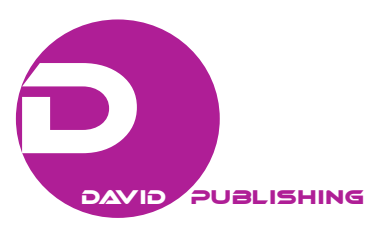

\title{
Implementing the SAS Joint Data Mining Certificate at Bryant University
}

\author{
Billie Anderson, Phyllis Schumacher and Alan Olinsky \\ Bryant University, Smithfield, RI
}

\begin{abstract}
Bryant University is currently one of only 18 schools worldwide that offers a SAS Joint Data Mining Certificate. With the availability of very large data sets, Data Mining has become an important quantitative skill to have in business and other fields. Bryant's program was successfully completed by 22 undergraduate students last year. This paper provides a description of Bryant's program along with the history of how the program was started and how it has grown. We discuss the curriculum, teaching materials, the SAS collaboration and some of the challenges which have been encountered.
\end{abstract}

Keywords: SAS programming, data mining, design of experiments

\section{Introduction}

Today's data-driven society means that statistical educators must prepare our students to compete in an environment where there is an explosion of data available to organizations. No matter what industry a student chooses to work in today, the prospective data analyst/programmer/statistician will be flooded with data and needs skills that readily make him or her capable to work with quantitative information (Davenport \& Kim, 2013). A recent McKinsey Global Institute report cited that by 2018 the United States could face a shortage of 140,000 to 190,000 with deep analytical skills as well as 1.5 million managers with the know-how to use analytical reports and analyses to make decisions (Manyika, et al., 2011).

The skills that a student needs to be successful at collecting, analyzing, and understanding data come from disciplines such as computer science, mathematics, statistics, engineering, and physics. The company Kaggle organizes data competitions in which anyone can enter and compete for the best solution challenging data analysis problems. Currently, one of the best ranked competitors on kaggle.com is an actuary that works in Singapore. Bryant University is committed to responding to the job market's needs by offering specialized classes in working with data sets, both small and large, and turning that data into meaningful decision-making results.

Bryant University has partnered with SAS, a leading provider of statistical software, to offer students a joint certificate in data mining. Data mining allows the searching of these datasets for important patterns and is used extensively in predictive modeling (Hand, et al., 2001). Using SAS as a teaching resource in a higher education environment has proven to be a valuable asset for the Mathematics Department at Bryant University.

Corresponding author: Billie Anderson, Ph.D., Assistant Professor, Mathematics Department, Bryant University. Phyllis Schumacher, Ph.D., Professor, Mathematics Department, Bryant University. Alan Olinsky, Ph.D., Professor, Mathematics Department, Bryant University. 
Students who complete a four course sequence of classes in statistics receive a joint certificate from Bryant and SAS. Certification is valuable to both undergraduate and graduate students. We know that many of our students have been able to obtain internships and full time positions because of their completion of our certification program at the undergraduate level. At the graduate level, professionals from diverse fields may enhance their quantitative credentials by obtaining a SAS certificate in data mining. Bryant has found that offering a SAS certification program to students gives them a competitive differentiator as they compete in an exploding analytical job market.

In section 2 the authors present the history of the SAS Joint Data Mining Certification. A high level overview of the curriculum is given in Section 3. In addition the authors describe three of the classes in more detail. Section 4 describes the SAS education philanthropy and the teaching materials that SAS makes available to higher education. The authors describe which of the SAS teaching tools the SAS Data Mining Certification courses employ as well as discuss additional teaching materials needed in an academic classroom. Section 5 showcases some of the student successes that have been achieved due to the certification that Bryant University offers. The implementation of this program has not been without challenges and those challenges are outlined in Section 6. Finally, Section 7 concludes with the authors looking forward to the future of the SAS certification program and discusses plans to grow the certification into a graduate certification process.

\section{History}

The SAS Joint Data Mining Certificate program is offered by the Mathematics Department in the College of Arts and Sciences at Bryant. The Mathematics Department was originally a department in the College of Business initially offering required mathematics courses in quantitative methods and statistics for business majors, and several electives including a second applied statistics course and graduate level quantitative business courses. In the seventies, the department offerings were increased to include other electives and also to offer a minor in both Mathematics and Statistics. In the early 80s a major in Actuarial Mathematics was established. This major has been very successful and has grown from its first graduating class in 1986 of 5 students to its current 172 students. Subsequent to the establishment of this major, in 2001, Bryant College became Bryant University and the mathematics department became part of the newly established College of Arts and Sciences. In 2007, an Applied Math/Stat major was introduced in response to demand from current and potential student inquiries. As the programs grew, the demand for electives grew as well. At the same time, Professor Alan Olinsky attended a summer SAS training program in Data Mining and introduced a course in Data Mining using SAS. This course was followed shortly by a course in SAS programming (these courses are required by SAS for joint certification). The courses became very popular and eventually led to the establishment of the current SAS Joint Data Mining Certificate program. The program is offered at the undergraduate level and is completed by Actuarial Mathematics (AM) majors, Applied Mathematics and Statistics (AMS) majors as well as Accounting, Finance, Marketing, Management, Psychology, and other liberal arts and business majors. This past year, 22 undergraduate students earned SAS certification in Data Mining.

We are in the final stages of offering a similar certificate at the graduate level. We know that this program will appeal to students since it has already been a huge success at the undergraduate level. This history is important to indicate the range of students that are currently enrolled in our program and also to differentiate our program from many of the programs that are geared to strictly business majors. 


\section{The Curriculum}

The curriculum for this program has been designed in partnership with SAS, a leading provider of data mining and business intelligence software and services. Currently, Bryant's undergraduate certification program is one of only 18 universities worldwide authorized to grant a joint certificate in data mining with SAS. These programs are both graduate and undergraduate programs.

Certification is awarded upon completion of four of the following 400 level courses.

Three required courses:

\section{M455 SAS Programming and Applied Statistics}

This course provides an introduction to SAS programming and covers the material required for the SAS Base Programming Exam. The first part of this course focuses on the following key areas: reading raw data files and SAS data sets; investigating and summarizing data by generating frequency tables and descriptive statistics; creating SAS variables and recoding data values; sub setting data; combining multiple SAS files; creating listing, summary, HTML, and graph reports. The second part of this course focuses on how to manage SAS data set input and output, work with different data types, and manipulate data. Specifically, this part of the course discusses using the DATA step to control SAS data set input and output, combine SAS data sets, summarize data, process data iteratively with DO loops and arrays, and perform data manipulations and transformations. This course is required for our joint certificate with SAS in data mining. A major programming project will be required.

\section{M460 Applied Data Mining}

Employing SAS Enterprise Miner software with real-world case studies, this course introduces students to the current theories, practices, statistical tools and techniques in "data mining," which embodies cutting-edge methods to reveal competitive insight, market advantage, and strategic opportunities. This course will cover the most useful statistical tools in data mining such as cluster analysis, logistic regression, classification trees, and neural networks. In addition, a comprehensive real-world data project will be required along with a presentation to the class and other interested parties of key aspects of the project with an analysis of the results.

\section{M461 Applied Multivariate Statistics}

After a brief review of multiple regression and analysis of variance, students are introduced to multivariate statistical techniques including principal components analysis, factor analysis, cluster analysis, discriminant analysis, logistic regression and multivariate analysis of variance. Although this course will not be theoretical, it will provide an intuitive yet comprehensive discussion of each of these statistical methods. The computer package SAS will be used for analysis.

And one of the following two electives:

\section{M470 Statistical Design and Analysis of Experiments}

This course is an introduction to the design and analysis of statistical experiments. It will cover the main elements of statistical thinking in the context of experimental design and ANOVA. Students will learn to choose sound and suitable design structures and also how to explore real data sets using a variety of graphs and numerical methods and analyze these data sets from designed experiments and reach justifiable conclusions based on the analyses. This will be an applied course and will utilize SAS software for statistical analysis. 


\section{M475 Applied Analytics using SAS}

This course will include an in depth review of applied analytical approaches, challenges, and solutions. A hands-on approach will be emphasized throughout the semester. A brief review of analytical techniques will be included, as well as an introduction to further analytical tools such as multivariate analysis, predictive modeling, time series analysis and survey analysis. SAS Enterprise Guide Software will be introduced and utilized for applying hands on analysis to real world data problems.

Since the authors of this paper have expertise in SAS programming, data mining and design of experiments we now will provide more detail about Math 455, Math 460 and Math 470.

Math 455 is the SAS programming and Applied Analytics course. How to teach an undergraduate student who has never programmed in the SAS language and relate those programming concepts to applied statistics problems can be challenging. Two concepts used in the classroom to help students learn the SAS programming language is to foster discovery and practice (Koft, 2006). The discovery part of the course is inherently in the makeup of the class. Typically, the students in the class are mathematics majors, finance, accounting, or some other quantitatively based major. These students are curious about statistics and mathematics and have a desire to understand how code can develop statistical applications.

The other aspect of teaching a programming course is to give the students plenty of practice. In class, students are explained a statistical concept and then showed how to implement that statistical concept in SAS code. Then, the students are given multiple homework assignments that reinforce programming concept shown in class. The programming homework that is assigned is related to applied statistical problems discussed in class. A credit score example is given in the next few paragraphs.

The Kolmogorov-Smirnoff (KS) statistic and the Gini statistic are used in many banks and financial institutions to discriminate between good and bad scorecards (Siddiqi, 2006). The KS statistic is used to determine what the cutoff credit score should be for credit applicants to be accepted or rejected for a loan. The KS statistic measures the maximum point of separation between the cumulative distribution functions between the good and bad credit loan applications. The credit score in which this maximum separation occurs is the credit score that a financial institution uses to determine which applicants to accept for credit and which ones to reject.

The Gini statistic is a particularly nice statistical programming application to use in a SAS programming course to illustrate an applied statistical concept because the Gini statistic is related to two statistical measures that the students would have been exposed to other classes. The Gini statistic is mathematically equivalent to the Mann-Whitney Wilcoxon statistic and is equivalent to the area under a receiver operating characteristic curve (ROC) curve (Hand, 2005). The students could have possibly been exposed to the Mann-Whitney Wilcoxon statistic in another upper level statistics course and the ROC curve is studied in Math 470 when model evaluation is discussed. The students learn that in an applied statistical setting the Gini statistic is used to determine that probability that a randomly chosen good credit applicant will have a higher credit score than a randomly chosen bad credit applicant.

The students are shown how to calculate the Gini and KS statistics using a data set with 10 data points. A homework assignment given to the students is to calculate the Gini and KS statistics on a data set that is much more realistic in terms of what a credit card company would have. The data set used in the assignment has 100,000 observations. In addition, the students are given a handout to read that relates the mathematical 
relationship between the KS and the Gini statistics and the students must create code that mimics the relationship. The code that the students develop for this assignment uses many aspects of the class such as manipulating data, performing do loops, and making transformations of variables.

Math 460 is the data mining class. The focus of data mining is different from traditional statistics. For example, in data mining one is not concerned with statistical inference such as hypothesis testing. Data mining is characterized by its ability to process a large amount of information. The large of amount of information is often found in corporate databases that are the result of collecting transactional data. Data mining has two goals: build predictive models and find useful and informative patterns in large amounts of data. The first assignment that the students are given is to find a journal article that describes the differences between data mining and traditional statistics. Each student is required to address the class and state the article they found and describe some of the differences their article points to as the difference between data mining and traditional statistics.

The three predictive models that are covered in the course are decision trees, logistic regression, and neural networks. The mathematical details of each of these models is discussed and explained in class. For decision trees, a mock data set is given with two variables and the students have to create the first 5 splits in the decision tree by hand. The likelihood function of logistic regression is derived and a sketch of the Newton-Raphson algorithm is given. Neural networks are motivated using nonparametric regressions. Once nonparametric regression is explained the limitations of nonparametric regressions are given. The neural networks are a nice alternative to nonparametric regressions because a specific functional form does not have to be specified.

Throughout the course homework assignments and case studies are assigned that are very similar to the demonstrations shown in class. Each case study requires the student to summarize the problem at hand, analyze the data, provide results, and explain the results as though they are talking to a non-statistician. The case studies are an attempt to allow the students to explain technical material in a non-technical manner that mimics the responsibility of a real world data miner.

The final project and presentation consists of taking a real-world data mining problem and producing a solution. There are two main components to the final project; a two page executive memo and a 20 minute presentation. The students are divided into groups of 3 to 4 people. A group presentation is part of the requirement from SAS in order for the students to receive the SAS Joint Data Mining certification.

Math 470 is the statistical design and analysis of experiments class. While the importance of analyzing large and complex data sets are discussed in classes such as Math 460. The Bryant University curriculum realizes that not all data sets that an applied statistician will analyze in his or his or her career will be extremely large. Math 470 deals largely with data that has not been developed from a well-developed experiment. Much of the data in a data mining application is the result of transactional or web-based data; that is an observational study. An observational study consists of the collection and analysis of data without changing any conditions. The purpose of a design of experiments class is to teach the students about experimentation.

An experiment deliberately imposes a treatment on a group of subjects or objects and the end goal is to observe a response. The validity of an experiment is directly affected by its construction and execution, attention to experimental design is extremely important. This importance for the design of experiments stems from the statisticians viewpoint of trying to determine relationships and inference as opposed to just describing what is being observed. Statisticians are rarely satisfied with only describing events they observe. Statisticians want to make inferences about what produced or contributed to a certain response. In order to get to the root 
cause of an event or response, an experimental design is required. An experimental design allows the statistician to rule out ambiguous effects that affect the response and focus on the relationships that produced the response.

There are several classic designs that are covered in Math 470. The first is randomization. The designs include randomized block designs, repeated measures, and a mixed model design. Examples of each design are given in class for the students. A classic example of a randomized block design that is commonly used is a researcher carrying out a study of the effectiveness of four different skin creams for the treatment of a certain skin disease. The researcher has eighty subjects and plans to divide them into four treatment groups of twenty subjects each. Using a randomized block design, the subjects are assessed and put in blocks of four according to how severe their skin condition is; the four most severe cases are the first block, the next four most severe cases are the second block, and so on to the twentieth block. The four members of each block are then randomly assigned, one to each of the four treatment groups.

Rather than a single class project in Math 470 for which students would take the semester to design, run and analyze an experiment, the class is set up with a series of smaller case like projects which allow the students to learn to use SAS to analyze experiments with many different designs, some of which are quite complicated, and provide a well written report of the results.

The first assignment in the class, which is assigned before learning how to analyze an experiment but after learning the terminology and assumptions for various designs, is to do a web search to find in the literature an application of experimental design, which has a single response variable and two or more independent variables. The students must then write a one to two page report describing the experiment. Within the report, they must identify the type of design and the identification of the dependent variable the independent variables along with their levels. The students must describe the statistical results in clear and readable report utilizing the American Psychological Association style.

After the experiment report assignment, as each of the various designs are discussed in class, the students are assigned selected problems from the text for which they are to completely analyze a data set including checking for all assumptions using SAS Enterprise Guide and provide a well written executive summary of the results.

The final assignment of the semester is a three to five page research paper and presentation of the description of a research question which is answered by a designed experiment. This report must include a data set and they must obtain the necessary output using SAS Enterprise Guide.

\section{Teaching Materials}

SAS CEO Jim Goodnight believes that education is the engine of economic growth. SAS has an education philanthropy (SAS Institute, 2012). This philanthropy provides teaching materials to help professors incorporate SAS into their teaching, including presentation slides, data sets and printable/editable files of the student manual. These materials are provided free of charge, with copyright permission for professors to use any of the content in their own class notes.

SAS also provides the software used in the classroom for no cost to students or professors. SAS OnDemand for Academics provides an online delivery model for teaching and learning data management and analytics. By connecting to a SAS-hosted server over the Web, users access the analytical power of SAS software through a user-friendly, point-and-click interface. 
Since we are in a higher education environment it is vital that we have an appropriate academic textbook to accompany the SAS software. With the advancement of software it is very easy for a student to produce output from a very complex statistical analysis. Without the appropriate academic training a student will not know the statistical methodology behind the output, what the output means, how to interpret the output, and the common pitfalls that need to be avoided based on the type of statistical analysis being performed. For example, some pitfalls that often occur with a non-academically trained analyst is not knowing and assaying the data properly. That is, not having an intimate knowledge of the data. At a very basic level, a student must know how to deal with missing values and outliers and how each of these types of issues can affect the model. For instance, a decision tree can naturally handle missing values whereas a logistic regression cannot. Logistic regression requires action to be taken on the missing values or the analyst can produce an unexpected model. As educators in statistical higher education we are constantly seeking how to find an appropriate balance of giving students a solid statistical background with an appropriate academic textbook while ensuring the students have appropriate access to state-of-the-art software and technology in order to stay competitive.

In previous statistical design of analysis classes, JMP software has been used with the use of an academic textbook and SAS teaching materials. JMP is statistical software created by SAS. Pronounced as "Jump", JMP is a tool for exploring data and interacting with it. JMP offers capabilities for design and analysis in a form a student can easily use. JMP allows the student to dynamically link data and graphics allowing the students to actively manipulate factors according to a specified design. JMP is the preferred statistical software package for the Math 470 class. The textbook book chosen was changed because the instructor wanted to have JMP output and results in the text to help students with running advanced topics in JMP. Currently, the Math 470 class is using SAS Enterprise Guide for the software piece of the class. The reason for choosing this software package is the availability of a pedagogical textbook that has Enterprise Guide output. (Gamst, Myers, \& Guarino, 2008).

\section{Student Successes}

Many of our graduates have reported that having SAS certification has greatly helped them not only in obtaining a job but also in advancing in their field. We want to highlight two success stories from among our first certification recipients. One of the first students to complete SAS certification was an AM major who first used SAS for a large accounting firm working on consulting projects for the federal government. He went on to several other positions, all requiring the use of SAS and each with a significant salary increase. Most recently, he moved to a position with the federal government utilizing SAS in a UNIX environment. He also is currently teaching an introductory SAS programming class at a large university.

Another of our first certification recipients recently contacted us to inform us that she had returned to graduate school and she feels that her SAS training is extremely helpful. She will be taking a course titled "Using Large Databases" next semester. She also felt that having knowledge of SAS was extremely helpful in getting her first job out of college. In her professional work, she dealt with national survey, assessment and administrative data and she expressed that the data mining background helped her to, in her words, "explore data to answer tough research questions". This led her to her current graduate work which requires the analysis of data in relationship to educational policy. To further quote this alumna, "The Data Mining certificate was a vital factor getting my foot through the door and exploring exciting career and education paths I did not even know existed." 


\section{Challenges}

There are a number of challenges that we have encountered as the SAS certification program has increased in popularity. The popularity among non-math majors to obtain the SAS certification has been steadily increasing. Two of the four classes are at full capacity for the Fall 2012 semester. It can be difficult to explain to students with non-quantitative backgrounds the intricacies and statistical details of data mining algorithms and designed experiments. Bryant is currently considering making the requirements to enter the four courses required for the certification more rigorous.

While SAS OnDemand is a wonderful piece of technology it does frequently crash and freeze up when many students are using it at the same time in class. Also, students may be working on their local computer and not realize that they have been disconnected from the server at SAS and so they are not able to save their latest work.

The data mining class has started to move away from using SAS OnDemand and is currently in a pilot program sponsored by the Bryant IT department that allows students to access SAS software on virtual machines. In addition, SAS does provide a classroom license that includes SAS, Enterprise Miner, and Enterprise Guide at a very reasonable cost. SAS is also now beta testing a web based interface for their SAS language. It would be expected that this would also be provided through the OnDemand program and be free of charge for students and instructors.

\section{Discussion}

SAS Joint Data Mining Certificate program has been extremely successful at Bryant University. While there are a few challenges as outlined above, they are greatly outweighed by the rewards.

Due to the success of the undergraduate program, the Math department is currently developing a graduate SAS certification program. This will allow working professionals to obtain a graduate level SAS Joint Data Mining Certification. Developing and teaching the courses for the SAS Joint Data Mining Certificate program not only offers the students a competitive advantage but also allows the faculty who teach the courses the ability to stay up-to-date on current statistical technology and teach with materials other than a textbook and chalkboard. By using SAS software and technology in the classroom we are able to bring new life to the world of statistics. We are able to teach tried and true statistical methods such as logistic regression and factorial designs with a 'twist' by being able to produce graphs and output that would not be possible in a traditional classroom setting. Not only does the software bring life to the data and make it easier to tell a story and make decisions with data, it allows the students to have a substantive credential on their resume that will allow them to compete in an analytical job marketplace that shows no signs of slowing down.

\section{References}

T. Davenport, J. Kim, Keeping up with the quants, Boston, MA: Harvard Business School Publishing, (2013).

J. Manyika, M. Chui, B. Brown, J. Bughin, R. Dobbs, C. Roxburgh, A. H. Byers, Big data: The next frontier for innovation, competition, and productivity report, New York, NY: McKinsey Global Institute, (2011).

P. Olson, World's Top Data Scientists Open Doors to Big-Cash Contests retrieved on 13 August 2013 from, (2012).

D. Hand, H. Mannila, P. Smyth, Principles of data mining. Cambridge, MA: MIT Press, (2001).

J. Koft, How to grow a SAS programmer, Proceedings of the $31^{\text {st }}$ SAS Users Group International Conference, San Francisco, USA, (2006), 1-3. 
N. Siddiqi, Credit risk scorecards: Developing and implementing intelligent credit scoring, Hoboken, NJ: John Wiley \& Sons (2006).

D. J. Hand, Good practice in retail credit scorecard assessment, The Journal of the Operational Research Society, 56(9) (2005) $1109-1117$.

D. J. Hand, Data mining: statistics and more?, American Statistician, 52(2) (1998) 112-118.

P. D. Allison, Logistic regression using SAS: Theory and application, Cary, NC: SAS Press (2012).

B. D. Ripley, Pattern recognition and neural networks, New York, NY: Cambridge University Press (2008).

V. J. Easton, J. H. Mccoll, Statistics Glossary, The STEPS Project, (1997). SAS Institute, (2012), Corporate Sustainability Report, Retrieved August 08, 2013. http://www.sas.com/company/csr_reports/archive/csr-report-current.pdf

G. Gamst, L.S. Myers, A.j. Guarino, Analysis of variance designs: A conceptual computational approach with SPSS and SAS, New York, NY: Cambridge University Press, (2008). 